ARTICLE

\title{
Diurnal $\mathrm{Fe}(\mathrm{II}) / \mathrm{Fe}(\mathrm{III})$ cycling and enhanced $\mathrm{O}_{2}$ production in a simulated Archean marine oxygen oasis
}

\author{
A. J. Herrmann (10 ${ }^{1}$, J. Sorwat ${ }^{2}$, J. M. Byrne ${ }^{2}$, N. Frankenberg-Dinkel ${ }^{1} \&$ M. M. Gehringer (1) ${ }^{1 凶}$
}

The oxygenation of early Earth's atmosphere during the Great Oxidation Event, is generally accepted to have been caused by oceanic Cyanobacterial oxygenic photosynthesis. Recent studies suggest that $\mathrm{Fe}(\mathrm{II})$ toxicity delayed the Cyanobacterial expansion necessary for the GOE. This study investigates the effects of Fe(II) on two Cyanobacteria, Pseudanabaena sp. PCC7367 and Synechococcus sp. PCC7336, in a simulated shallow-water marine Archean environment. A similar Fe(II) toxicity response was observed as reported for closed batch cultures. This toxicity was not observed in cultures provided with continuous gaseous exchange that showed significantly shorter doubling times than the closed-culture system, even with repeated nocturnal addition of Fe(II) for 12 days. The green rust (GR) formed under high Fe(II) conditions, was not found to be directly toxic to Pseudanabaena sp. PCC7367. In summary, we present evidence of diurnal Fe cycling in a simulated shallow-water marine environment for two ancestral strains of Cyanobacteria, with increased $\mathrm{O}_{2}$ production under anoxic conditions.

\footnotetext{
${ }^{1}$ Department of Microbiology, Technische Universität Kaiserslautern, 67663 Kaiserslautern, Germany. ${ }^{2}$ Geomicrobiology, Centre for Applied Geosciences, University of Tübingen, 72074 Tübingen, Germany. ${ }^{凶}$ email: mgehring@bio.uni-kl.de
} 
$\mathrm{T}$ he start of the Great Oxidation Event (GOE) 2.43 Ga (billion years ago $)^{1}$ marked the beginning of the Proterozoic Eon and initiated the permanent oxygenation of Earth's atmosphere and oceans ${ }^{2}$. Although the GOE is associated with the widespread release of oxygen presumably by oxygenic phototrophic Cyanobacteria ${ }^{3}$, the rock record shows indications of oxygenic photosynthesis $200 \mathrm{Myr}$ (million years) prior, in the form of tufted microbial mats and stromatolites in the $2.7 \mathrm{Ga}$ Tumbiana Formation, West Australia ${ }^{4,5}$. Potential indications of phototrophic mats with Cyanobacterial tufted mat morphology have been identified in the widespread shallow tidal mats in the 3.2 Gyr (billion years) old Moodies Group, South Africa, which are thought to be phototrophic 6,7 . Geochemical markers that reflect transient or local occurrences of low levels of oxygen are also indicators for oxygenic photosynthesis prior to the GOE at 2.8-3.0 $\mathrm{Gyr}^{8}, 9$. Many hypotheses exist as to why the occurrence of oxygenic photosynthesis and the timing of the GOE are temporally uncoupled, including the consumption of released oxygen by redox-sensitive elements, such as sulphur, or cell respiration ${ }^{3}$, the time needed to adapt to a pelagic life style necessary to produce $\mathrm{O}_{2}$ on a large scale ${ }^{10,11}$ or the toxicity of an anoxic, ferruginous Archaean ocean ${ }^{12}$. All of these proposed processes may have contributed towards restricting the expansion of Cyanobacteria to a few highly productive niches, thereby generating the occasional 'oxygen oases' recorded in the rock record ${ }^{13}, 14$. These localised hot-spots bearing up to $10 \mu \mathrm{M}$ dissolved oxygen ${ }^{14}$ would have mitigated the reported toxic effects of $\mathrm{Fe}(\mathrm{II})$ due to oxidation to insoluble $\mathrm{Fe}(\mathrm{III})^{12}$. $\mathrm{Fe}(\mathrm{III})$ in turn could have played a major role in the formation of banded iron formations (BIF), either directly as $\mathrm{Fe}(\mathrm{III})$ oxides $^{15,16}$ or as green rust (GR), after reacting with locally remaining $\mathrm{Fe}(\mathrm{II})^{17}$. The localised concentration of oxygenic phototrophs may have resulted in weathering of nearby rock surfaces, releasing essential micronutrients, such as sulphates and molybdenum ${ }^{18}$. By combining these hypotheses, a graphical representation of an Archaean oxygen oasis was created (Fig. 1) to illustrate diurnal Fe(II)/(III) fluxes.

Simulations of the processes related to Archaean 'oxygen oases' under today's atmosphere in small-scale laboratory conditions remain challenging and are conducted either in 'closed' bottle systems $^{12,} 19$ or 'open' anoxic workstations ${ }^{20}$. Differences in experimental setups and Cyanobacterial strains used make it difficult to obtain a clear overview of Fe(II) toxicity and its effects on photosynthesis in ancestral Cyanobacterial strains. Cyanobacteria are the only bacteria that possess a circadian clock that regulates, amongst others, iron uptake and the redox status within the cell ${ }^{21}$, making it essential to conduct experiments on Archaean $\mathrm{Fe}(\mathrm{II})$ toxicity under diurnal conditions. Here we directly compare two culture systems by setting up otherwise identical experiments to determine the reported toxicity of $\mathrm{Fe}(\mathrm{II})$ on two basal clade, saltwater Cyanobacteria strains, the multicellular Pseudanabaena sp. PCC7367 and unicellular Synechococcus PCC7336. The simpler closed-culture system utilises a sealed container with a static initial atmosphere with no gaseous exchange. The open-culture system permits continual gaseous exchange with the larger atmospheric reservoir of an anoxic workstation, which removes $\mathrm{O}_{2}$ and replenishes $\mathrm{CO}_{2}$ in the atmosphere. The strains were chosen because they are the mostprimitive recent saltwater Cyanobacteria, with a close phylogenetic relationship to the root Cyanobacteria, Gloeobacter violaceus sp. PCC742111, 22. The collected data for cell growth, Fe(II) oxidation and $\mathrm{O}_{2}$ production rates not only provide important constraints for further theoretical modelling, but also show the shortcomings of a closed growth system. After establishing an appropriate experimental setup for $\mathrm{Fe}(\mathrm{II})$ toxicity experiments, we explored the influence that repeated nocturnal addition of $\mathrm{Fe}$
(II) has on Cyanobacterial growth, in contrast to the single exposure experiments conducted so far. Of particular interest was the observation of GR formation during periods of low photosynthetic activity, when $\mathrm{Fe}(\mathrm{II})$ concentrations were high, as GR is thought to be an important contributor to the formation of $\mathrm{BIF}^{17}$.

\section{Results}

Iron toxicity in different growth systems. To assess $\mathrm{Fe}(\mathrm{II})$ toxicity and the influence of the experimental setup on growth, two marine basal clade Cyanobacteria, Pseudanabaena sp. PCC7367 and Synechococcus sp. PCC7336, were grown for 21 days with different $\mathrm{Fe}(\mathrm{II})$ starting concentrations approximating the proposed range of an Archaean ocean of 30-120 $\mu \mathrm{M}^{23}$. The growth systems were either closed bottles with a single injection of nitrogen with $10 \% \mathrm{CO}_{2}$ and $5 \% \mathrm{H}_{2}$ at the start of the experiment or an open system with ventilated culture flasks inside an anoxic chamber filled with forming gas $\left(\mathrm{N}_{2}\right.$ with $\left.\leq 5 \% \mathrm{H}_{2}\right)$ and constant removal of $\mathrm{O}_{2}$ while maintaining $0.2 \%$ of $\mathrm{CO}_{2}$, which is on the lower end of the proposed $\mathrm{CO}_{2}$ concentrations of an Archaean atmosphere 24,25 . Oxic control cultures were grown in parallel under normal atmospheric conditions in the open-culture system for comparison. The growth medium was buffered with $22 \mathrm{mM} \mathrm{NaHCO}_{3}$ to minimise the shift in media $\mathrm{pH}$ under different $\mathrm{CO}_{2}$ concentrations.

Monitoring of chlorophyll $a$ (Chl $a$ ) levels of photosynthetic organisms is a standard method for determining growth rates ${ }^{26}$. In the closed-culture system, Pseudanabaena sp. PCC7367 exhibits a significantly reduced Chl $a$ growth if $\mathrm{Fe}(\mathrm{II})$ is added at the start, with a doubling time of up to $\sim 20$ days in $600 \mu \mathrm{M} F$ (II) (Fig. 2a, e). In contrast, Pseudanabaena sp. PCC7367 in the anoxic open-culture system shows no significant difference in growth between the media controls and the cultures with $20 \mu \mathrm{M}$ $\mathrm{Fe}(\mathrm{II})$. Incubation with $120 \mu \mathrm{M} \mathrm{Fe}(\mathrm{II})$ resulted in a small, but significant, delay in Chl $a$ accumulation. Regardless of the culture system used, Fe(II) supplied at concentrations of $120 \mu \mathrm{M}$ or less was completely oxidised within 2 days (Fig. 2c). Oxidation of 600 $\mu \mathrm{M} \mathrm{Fe}(\mathrm{II})$, however, took up to 14 days in the closed-culture system, whereas in the open-culture system growth (Fig. 2a, b) Fe (II) oxidation (Fig. 2c, d) ceased after day 4. The slight increase in measured media $\mathrm{Fe}(\mathrm{II})$ at the end of stationary phase is most likely an artefact created by the incomplete removal of cell debris during the short centrifugation step to prevent $\mathrm{Fe}(\mathrm{II})$ oxidation during sample handling.

In the closed-culture system, Synechococcus PCC7336 demonstrated almost no growth, with a doubling time of $\sim 12$ days for the control and more than double that upon addition of Fe(II) up to 20 or $120 \mu \mathrm{M}$ (Fig. $2 \mathrm{~b}$, e). In the cultures provided with $600 \mu \mathrm{M}$ $\mathrm{Fe}(\mathrm{II})$ hardly any Chl $a$ growth was observed (doubling time of $>70$ days). Also, the oxidation of $\mathrm{Fe}(\mathrm{II})$ ceased after day 7, indicating low biological activity (Fig. 2d). In the anoxic openculture system Synechococcus PCC7336 control cultures grew rapidly, with a Chl $a$ growth approaching those of Pseudanabaena sp. PCC7367 anoxic control cultures (Fig. 2e). However, addition of $\mathrm{Fe}(\mathrm{II})$ up to 20 and $120 \mu \mathrm{M}$ increased the doubling times significantly $(p<0.05)$. Irrespective of which culture system was used, Fe(II) was completely oxidised after 2 days in cultures exposed to 20 and $120 \mu \mathrm{M}$ of $\mathrm{Fe}(\mathrm{II})$ (Fig. 2d). Cultures of Synechococcus sp. PCC7336 grown with $600 \mu \mathrm{M}$ Fe(II) in the open system showed a similar response to that seen for Pseudanabaena sp. PCC7367, with a cessation of biological activity after day 4 (Fig. 2b, d). At this point, the green precipitate, forming in the culture flasks of both strains, was collected and analysed by ${ }^{57} \mathrm{Fe}$ Mössbauer spectroscopy, which identified the substance as GR (Supplementary Fig. 7 and Supplementary Table 4). 

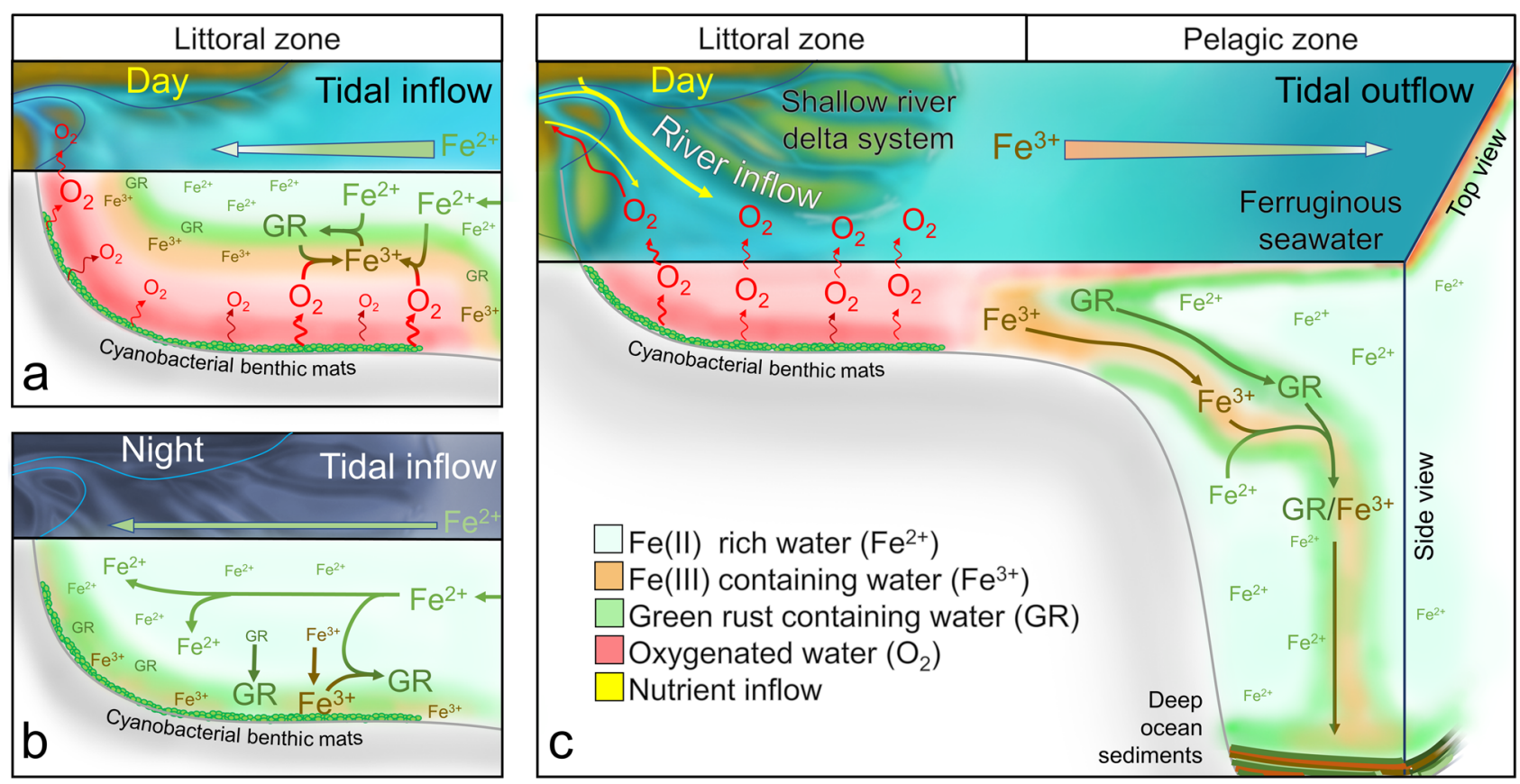

Fig. 1 Graphical model of a proposed Archaean littoral oxygen oasis. a A diurnal tidal inflow of ferruginous seawater creates an anoxic Fe(II) layer over oxygenic phototrophic Cyanobacterial mats. Photosynthetic oxygen creates green rust (GR) in the upper boundary layer by partial oxidation of Fe(II) during the day. Closer to the Cyanobacterial mats, Fe(III) is the dominant species, as Fe(II) and precipitating GR is completely oxidised. $\mathbf{b}$ During the night time, no oxygen is produced by photosynthesis and the littoral zone becomes ferruginous with precipitated Fe(III) oxides and/or GR, produced in the daytime, at the bottom layer. c Tidal outflow transports GR and Fe(III) in the anoxic open ocean, where the remaining Fe(III) can react with Fe(II) to GR. GR and/or Fe(III) sink to the bottom and form deep ocean sediments. High oxygen levels during the day oxidise Fe(II) from the pelagic zone and thereby prevent Fe(II) from reaching the littoral zone. Surplus oxygen not consumed by the oxidation of Fe(II) escapes into the atmosphere leading to localised land weathering, thereby releasing additional nutrients. Additional weathering at the oxygen-rich microbe-mineral interface cannot be excluded. Image created from references $6,12,13,17,18$.

In summary, the data show a highly significant $(p<0.005)$ decrease in Chl $a$ growth for both strains in the closed-culture vessels in comparison to the open-culture system at $\mathrm{Fe}(\mathrm{II})$ levels representing the Archaean ocean or lower (Fig. 2a, b, e). The open-culture system controls show no significant difference in Chl $a$ growth to the present atmosphere controls for the first 10 days, after which the open-culture system shows better growth (Fig. 2a, b).

Iron toxicity at daily exposure. To further expand on the experiments described above, which demonstrated that all $\mathrm{Fe}(\mathrm{II})$ in the concentration range proposed to have existed in the Archaean ocean was oxidised within 2 days, a second set of experiments were conducted to assess the effects of repeated exposure to $\mathrm{Fe}(\mathrm{II})$. In order to simulate a hypothetical reoccurring inflow of ferruginous Archaean seawater, Fe(II) was added to a concentration of $\sim 120 \mu \mathrm{M}$ every dark cycle for 12 days. The addition of $\mathrm{Fe}(\mathrm{II})$ was conducted once the $\mathrm{O}_{2}$ levels had dropped down close to zero due to cell respiration and passive gas exchange with the anoxic atmosphere in order to maximise the effect of $\mathrm{Fe}(\mathrm{II})$ exposure.

Repeated exposure to $\mathrm{Fe}(\mathrm{II})$ during the dark cycle over 21 days caused a significant $(p<0.05)$ drop in growth in both strains (Fig. 3a, b). Daily addition of Fe(II) leads to an increase in the doubling time of Pseudanabaena sp. PCC7367 by $\sim 50 \%$. Synechococcus PCC7336 was more sensitive to the prolonged Fe (II) exposure with $\mathrm{a} \sim 4 \mathrm{x}$ longer doubling time than the control culture. After day 4, the addition of $\mathrm{Fe}(\mathrm{II})$ during the dark cycle led to the temporary formation of a green precipitate, with an average $\mathrm{Fe}(\mathrm{II}) / \mathrm{Fe}$ (III) ratio of $2.54 \pm 0.025$, as determined by ferrozine assay for PCC7336, which is indicative of GR. During the first few hours of the light cycle, the precipitate was oxidised to a yellow/orange solid in the cultures of Pseudanabaena sp. PCC7367, while the oxidation took several days in the cultures of Synechococcus sp. PCC7336.

The Fe(II) retention experiment (Fig. 3d) showed that, in the absence of oxygenic photosynthesis, no Fe(II) oxidation occurs and cultures undergoing repeated $\mathrm{Fe}(\mathrm{II})$ exposure (Fig. 3a) were exposed to $\mathrm{Fe}(\mathrm{II})$ during the whole night cycle. The rapid decrease in dissolved $\mathrm{Fe}(\mathrm{II})$ levels, accompanied by an increase in the dissolved oxygen concentration, is only measurable after the start of the light cycle. The total iron levels measured $2 \mathrm{~h}$ after the start of the light cycle showed that almost all $\mathrm{Fe}(\mathrm{II})$ was oxidised. Repeating this experiment with stirred cultures of Pseudanabaena sp. PCC7367 demonstrated that even with increased release of oxygen into the anoxic atmosphere by rapid mixing at the water-air interface, all added $\mathrm{Fe}(\mathrm{II})$ was completely oxidised, although at a slower rate than observed in the standing cultures (Fig. 3c), while the solubilised oxygen concentration stabilised at $\sim 4.5 \mu \mathrm{M}$.

Toxicity of green rust. As in both previously described experiments, a strong reduction in growth was observed which coincided with the formation of GR. Experiments were therefore conducted to elucidate if GR is the cause of the cessation of biological activity, or merely a secondary by-product. Therefore, cultures of Pseudanabaena sp. PCC7367 were set up in the anoxic, open-culture system and their growth followed by Chl $a$ measurements. One set of cultures had $600 \mu \mathrm{M} \mathrm{Fe}(\mathrm{II})$ added at the start of the experiment, which would lead to the formation of GR as observed in the open-culturing experiment. At the same time the GR formation was observed in these cultures, GR was added to a parallel set of cultures during the dark cycle at levels 

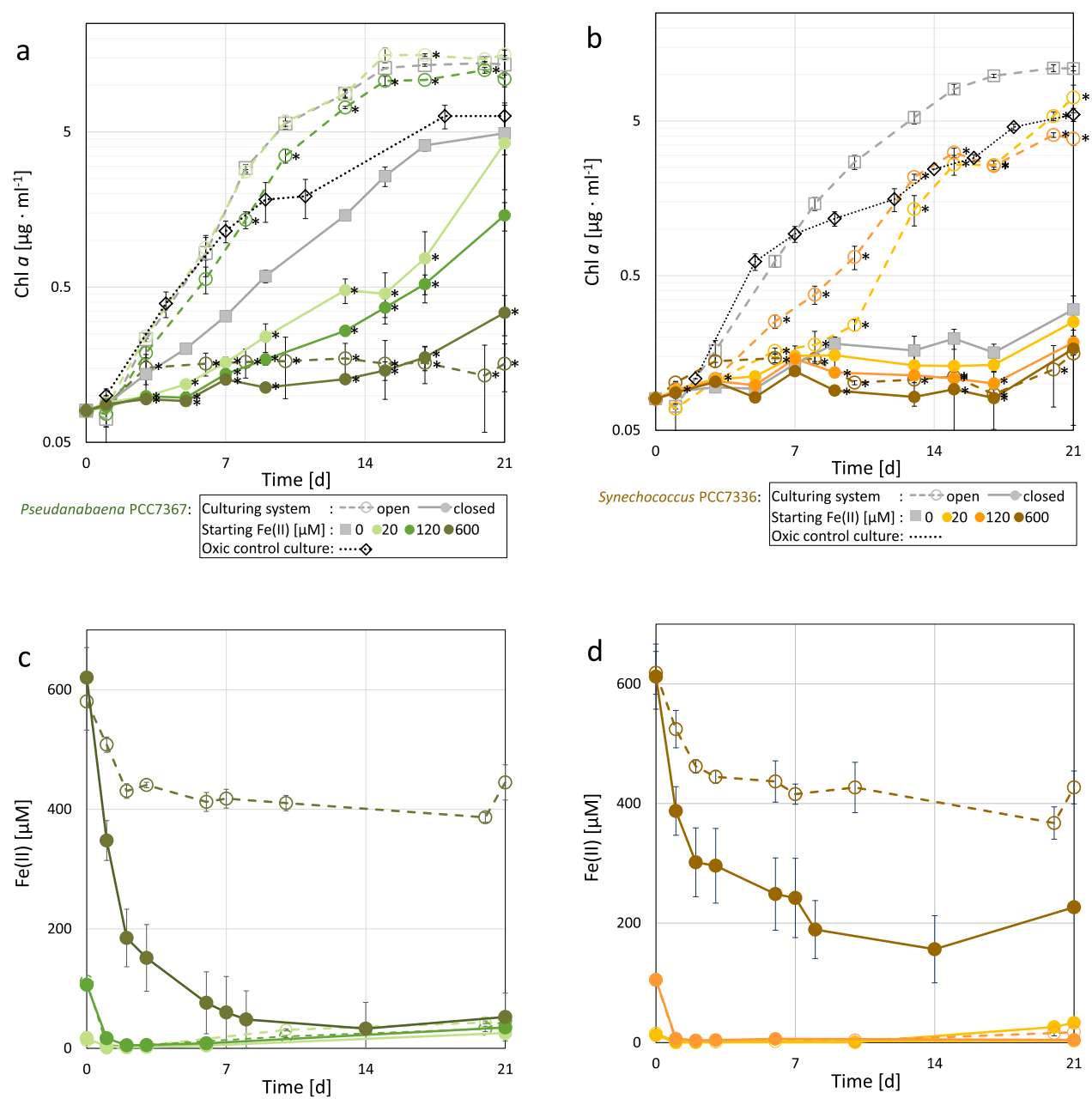

\section{e Doubling times during exponential growth [d]}

\begin{tabular}{|c|c|c|c|c|}
\hline Strain & \multicolumn{2}{|c|}{ Pseudanabaena sp. PCC7367 } & \multicolumn{2}{|c|}{ Synechococcus PCC7336 } \\
\hline Growth system & open & closed & open & closed \\
\hline Control & $1.4 \pm 0.11$ & $2.86 \pm 0.09$ & $1.72 \pm 0.05$ & $12.3 \pm 1.28$ \\
\hline $20 \mu \mathrm{M} \mathrm{Fe}(\mathrm{II})$ & $1.47 \pm 0.04$ & $4.56 \pm 1.33$ & $2.66 \pm 0.21$ & $27.16 \pm 4.59$ \\
\hline $120 \mu \mathrm{M}$ Fe(II) & $1.62 \pm 0.10$ & $6.62 \pm 0.95$ & $2.75 \pm 0.07$ & $32.17 \pm 11.54$ \\
\hline $600 \mu \mathrm{M} \mathrm{Fe}(\mathrm{II})$ & & & & \\
\hline $\begin{array}{l}d \leq 4 \\
d>4\end{array}$ & $\begin{array}{l}1.84 \pm 0.15 \\
-1\end{array}$ & $19.5 \pm 7.95$ & $2.99 \pm 1.41$ & $70.5 \pm 4.18$ \\
\hline Oxic control & $1.96 \pm 0.22$ & & $3.21 \pm 0.06$ & \\
\hline
\end{tabular}

Fig. 2 Illustration of the effects of the addition of different concentrations of Fe(II) on the growth of Pseudanabaena sp. PCC7367 and Synechococcus sp. 7336. Comparison of growth (a, b, e) and Fe(II) oxidation (c, d) of Pseudanabaena sp. PCC7367 (a, c) and Synechococcus sp. PCC7336 (b, d) between the open- and a closed-culture systems. The growth response of the two strains of saltwater Cyanobacteria in $22 \mathrm{mM} \mathrm{NaHCO}_{3}$-buffered $\mathrm{ASN}^{-I I I}$ media to different concentrations of $\mathrm{Fe}(\mathrm{II})$ was measured by $\mathrm{Chl}$ a determination every 2-3 days (a, b) for 21 days in closed anoxic culture bottles (solid lines) or open cell suspension flasks in an anoxic workstation (dashed lines). The Fe(II) starting concentrations were $20 \mu \mathrm{M}$ (PCC7367 light green/PCC7336 yellow), $120 \mu \mathrm{M}$ (PCC7367 green/PCC7336 orange) and $600 \mu \mathrm{M}$ (PCC7367 dark green/PCC7336 red). The controls (grey) were grown in ASN-III media with an $\mathrm{Fe}(\mathrm{III})$ concentration of $15 \mu \mathrm{M}$ under anoxic (solid/dashed line) and normal atmospheric conditions (dotted line). The oxidation of Fe(II) to Fe(III) was assessed in parallel using a spectrophotometric assay $(\mathbf{c}, \mathbf{d})$. Fe(II) measurements of abiotic controls are depicted in Supplementary Fig. 6. Cell doubling times (d) and their standard deviations during the exponential growth phase (e) were determined from day 1 to 13 for Pseudanabaena sp. PCC7367 and from day 1 to 17 for Synechococcus sp. PCC7336. The doubling time for the cultures with $600 \mu \mathrm{M} \mathrm{Fe}(\mathrm{II})$ in the open-culture system was split into a doubling time before green rust formation at day 4 and after. Data are presented as mean values \pm SD with $n=4$ biologically independent samples. Asterisks $\left(^{\star}\right)$ represent a significant difference to the media control grown in the equivalent growth system $(p<0.05$, Student's $t$-test, two-tailed). 


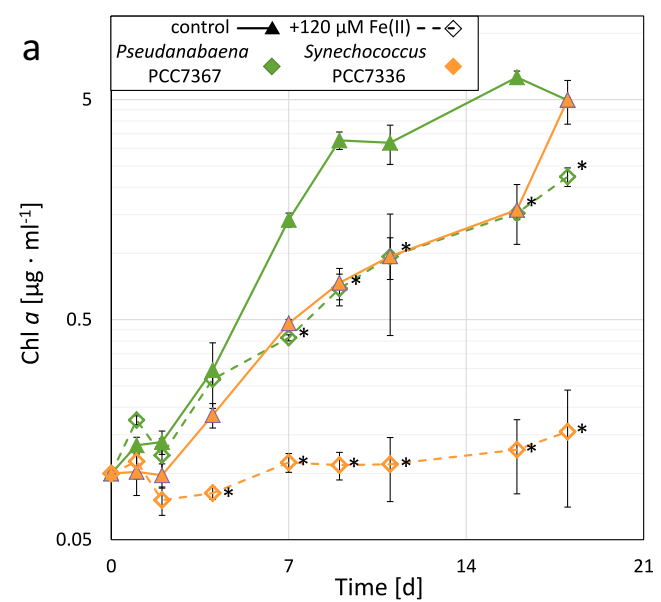

b Doubling times during exponential growth [d]

\begin{tabular}{l|l|l} 
Strain & $\begin{array}{l}\text { Pseudanabaena } \\
\text { sp. PCC7367 }\end{array}$ & $\begin{array}{l}\text { Synechococcus } \\
\text { PCC7336 }\end{array}$ \\
\hline Control & $1.49 \pm 0.06$ & $2.19 \pm 0.21$ \\
$120 \mu \mathrm{M} \mathrm{Fe}(\mathrm{II})$ & $2.83 \pm 0.38$ & $13.34 \pm 4.09$
\end{tabular}

C Fe(II) oxidation rates per $\mathrm{Chl} a\left[\mu \mathrm{mol} \cdot \mathrm{h}^{-1} \cdot \mathrm{mg}^{-1}\right]$

\begin{tabular}{l|l|l} 
Strain & $\begin{array}{l}\text { Pseudanabaena } \\
\text { sp. PCC7367 }\end{array}$ & $\begin{array}{l}\text { Synechococcus } \\
\text { PCC7336 }\end{array}$ \\
\hline standing & $41.24 \pm 4.59$ & $58.60 \pm 2.18$ \\
stirred & 26.46 & -
\end{tabular}

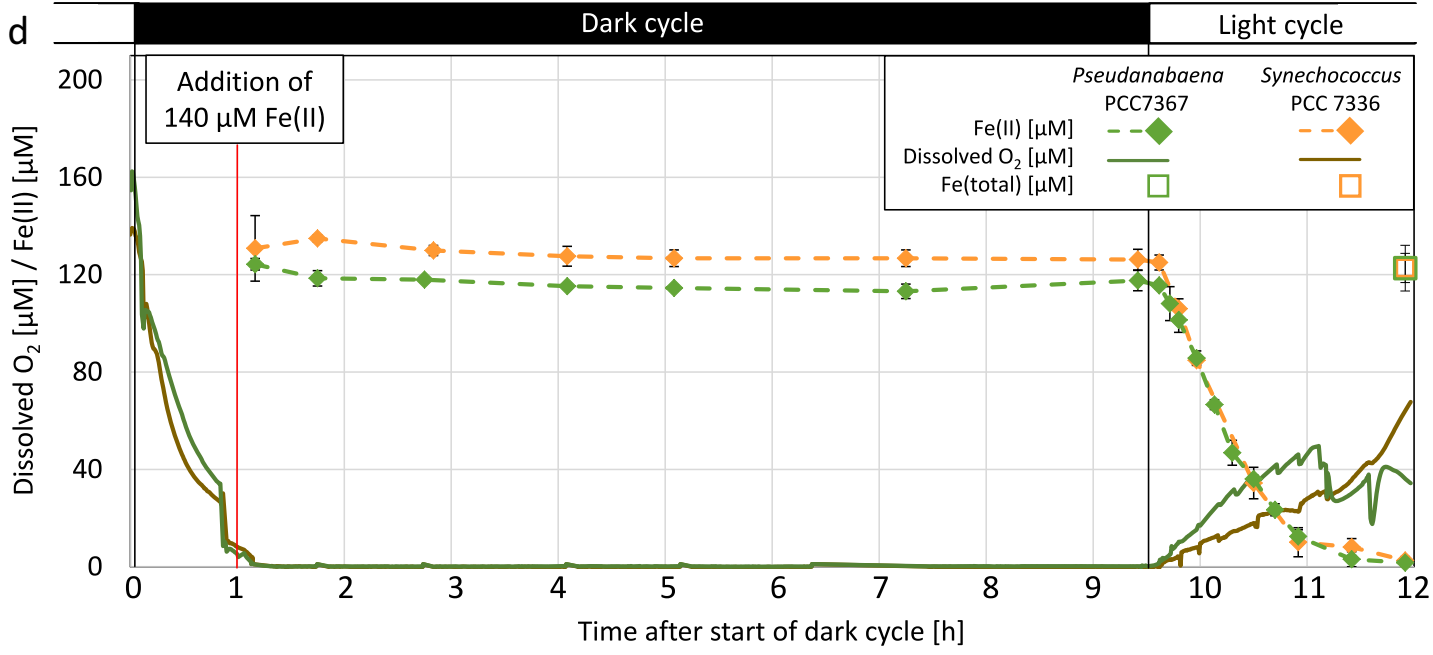

Fig. 3 The influence of reoccurring nightly addition of Fe(II) on the growth of Pseudanabaena sp. PCC7367 and Synechococcus sp. PCC7336. Both species were cultured in an open-culture system $(\mathbf{a}, \mathbf{b})$ and the oxidation of Fe(II) monitored $(\mathbf{c}, \mathbf{d})$. Chl $a$ growth curves (a) and doubling times (b) of the cultures in an anoxic open-culture system with a recurring nightly addition of $140 \mu \mathrm{M} \mathrm{Fe}(\mathrm{II})$. For the first 12 days during every dark cycle, the cultures were shaken briefly to release the dissolved oxygen and Fe(II) solution was added to a total concentration of 120-140 $\mu \mathrm{M}$. Chl a measurements (a) were taken every 2 nd to 3 rd day for 19 days. Cultures grown without Fe(II) addition were used as controls (solid line). Cell doubling times during the exponential growth (b) were determined from day 2 to 12 for both strains. The retention of Fe(II) during the dark cycle (d) was exemplary tested in analogous 7-day old, exponentially growing control cultures of Pseudanabaena sp. PCC7367 (green) and Synechococcus PCC7336 (orange) by measuring the Fe(II) concentration (dashed lines), while in parallel recording the oxygen concentration (solid line). The Fe(II) oxidation was measured every 10 min with a spectrophotometric ferrozine assay. Two hours after the start of the light cycle, the amount of Fe(III) in the media was assessed (open squares). The average rate of Fe(II) oxidation per $\mathrm{Chl} a$ during the light cycle (c) was calculated and compared to cultures which were additionally stirred at constant 250 rpm on a magnetic stirrer. Data are presented as mean values \pm SD with $n=4$ biologically independent samples, except for the stirred Fe(II) oxidation rate with $n=2$ biologically independent samples. Asterisks $\left(^{*}\right)$ represent a significant difference between Fe(II) supplemented culture and control $(p<0.05$, Student's $t$-test, two-tailed).

representing the GR levels observed in the section 'Toxicity assessment of green rust'.

The Chl $a$ growth curve (Fig. 4a) showed that the addition of GR on day 4 had no significant effect on growth in comparison to the negative control, regardless of concentrations used. The addition of $\mathrm{Fe}$ (II) on day 4 showed a small but significant growth retardation of the control culture. In contrast, the positive control, with $\mathrm{Fe}(\mathrm{II})$ added to a concentration of $600 \mu \mathrm{M}$ at the start of the experiment, showed a complete arrest of growth between days 4 and 7 until day 9. On day 9, growth could be restored by oxidising all remaining $\mathrm{Fe}(\mathrm{II})$ and GR to $\mathrm{Fe}(\mathrm{III})$ by the addition of ambient air for $10 \mathrm{~min}$ (Fig. 4a). Light microscopic pictures taken 2 days after the addition of Fe(II)/GR on day 6, showed intact filaments of Cyanobacteria attached to granules of yellow material with more or less dark green spots (Fig. 4d), relative to the amount of GR added 2 days prior. The dark green spots resemble the appearance of the abiotically produced GR (Fig. 4c), while the larger yellowish granules could be composed of Fe(III) compounds. The Cyanobacterial filaments and cells looked intact and appeared similar to the control culture without the addition of iron (Fig. 4b). The positive control cultures where GR was formed after Fe(II) addition, showed a similar yellowish granule structure with dark green spots (Fig. 4e) as seen in the samples where GR was added directly, however, very few intact Cyanobacteria cells were observed. In addition, structures resembling single Cyanobacteria cells encased in $\mathrm{Fe}(\mathrm{III})$ compounds (Fig. 4e, white arrows) were observed.

Oxygen production under anoxic conditions. As the production of oxygen by marine Cyanobacteria during the Archaean was likely the main driver of the GOE, the influence of an anoxic 

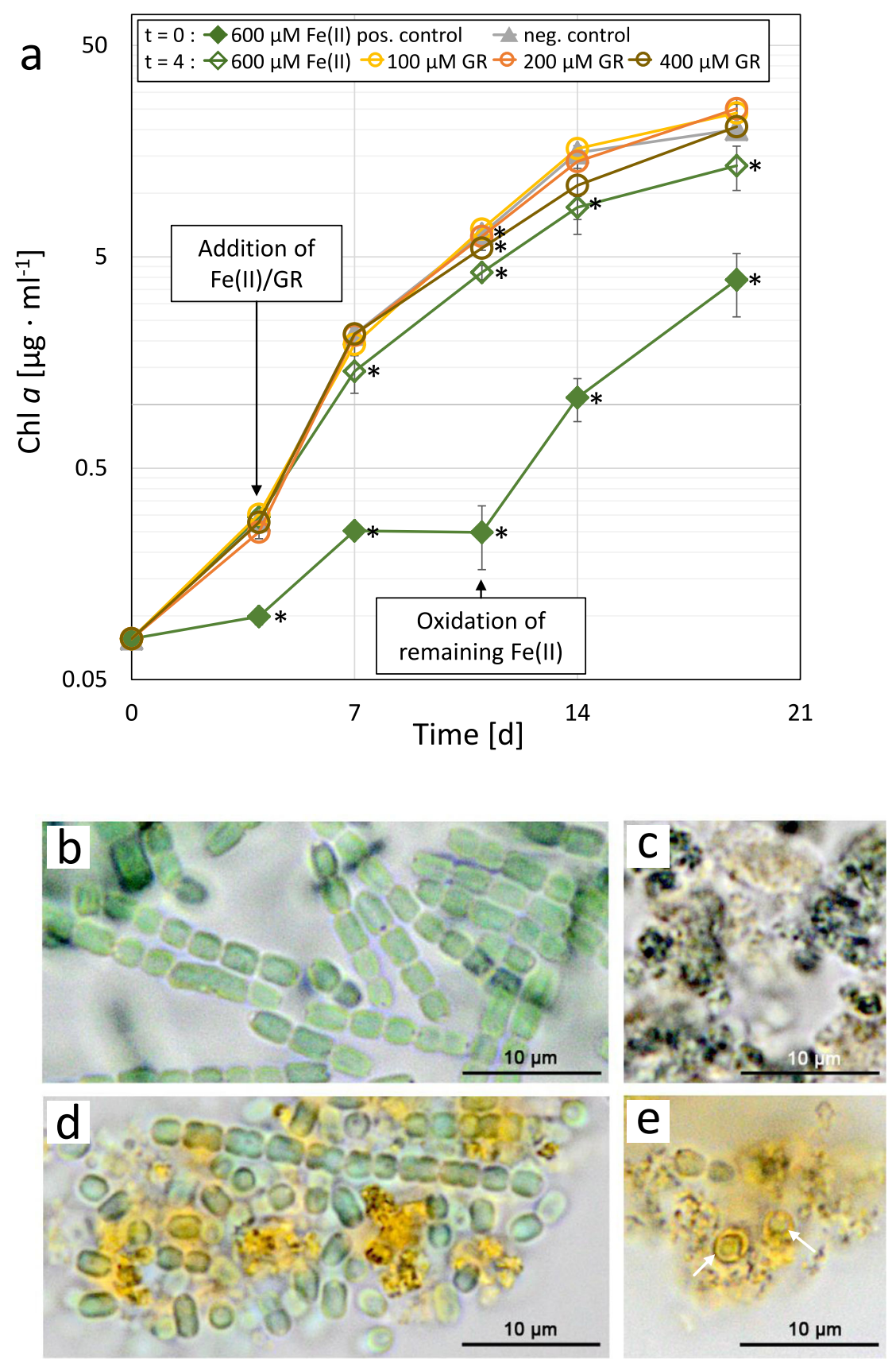

Fig. 4 Illustration of the effect of green rust on the growth of Pseudanabaena sp. PCC7367. The Chl a growth (a) and light microscopic pictures (b, d, e) of Pseudanabaena sp. PCC7367 cultures after the addition of Fe(II) or green rust (GR, c). Chl a growth curves (a) of cultures in an anoxic open-culture system with the addition of $600 \mu \mathrm{M} \mathrm{Fe}(\mathrm{II})$ (open, green), $100 \mu \mathrm{M} \mathrm{GR}$ (open, yellow), $200 \mu \mathrm{M}$ GR (open, orange) or $400 \mu \mathrm{M}$ GR (open brown) on day 4 of exponential growth. The positive control had $600 \mu \mathrm{M}$ Fe(II) added at the start of the experiment (closed, green), while the negative control had no addition of Fe (closed, grey). On day 6, 2 days after the addition of $\mathrm{Fe}(\mathrm{II}) / \mathrm{GR}$, light microscopic pictures were taken of the negative control culture (b), the GR in an abiotic control (c), the culture with the addition of $400 \mu \mathrm{M}$ GR on day 4 (d) and the positive control with $600 \mu \mathrm{M}$ Fe(II) at the start of the experiment (e). On day 9, the remaining Fe(II)/GR in the positive control was oxidised by exposure to oxic ambient air for 10 min. Data are presented as mean values \pm SD with $n=3$ biologically independent samples. Light microscopic images were taken at 100x magnification and are presented with a $10 \mu \mathrm{m}$ scale bar. Asterisks $\left(^{\star}\right)$ represent a significant difference between Fe(II)/GR supplemented cultures and the growth control $(p<0.05$, Student's $t$-test, two-tailed).

atmosphere on the photosynthetic production of oxygen was investigated.

Pseudanabaena sp. PCC7367, grown under anoxic conditions, showed a significant $(p<0.05)$ increase in net oxygen production per mg Chl $a$ in comparison to the cultures grown under modern $\mathrm{O}_{2}$ levels or elevated $\mathrm{CO}_{2}$ under the standard growth light intensity of 25 Photosynthetic Photon Flux Density (PPFD, $\mu \mathrm{mol}$ photons per $\mathrm{m}^{2} / \mathrm{s}$ ) (Fig. 5).
Measurements at 60 PPFD, which resembles $80 \%$ of the light intensity of highest measured net $\mathrm{O}_{2}$ production (Supplementary Fig. 8), still showed a significantly higher net $\mathrm{O}_{2}$ production under anoxic conditions over modern atmospheric growth conditions. The addition of $\mathrm{Fe}(\mathrm{II})$ at the start of the experiment, which was oxidised within 2 days, had no lasting, significant effect on the observed oxygen production rates, regardless of the test atmosphere. 


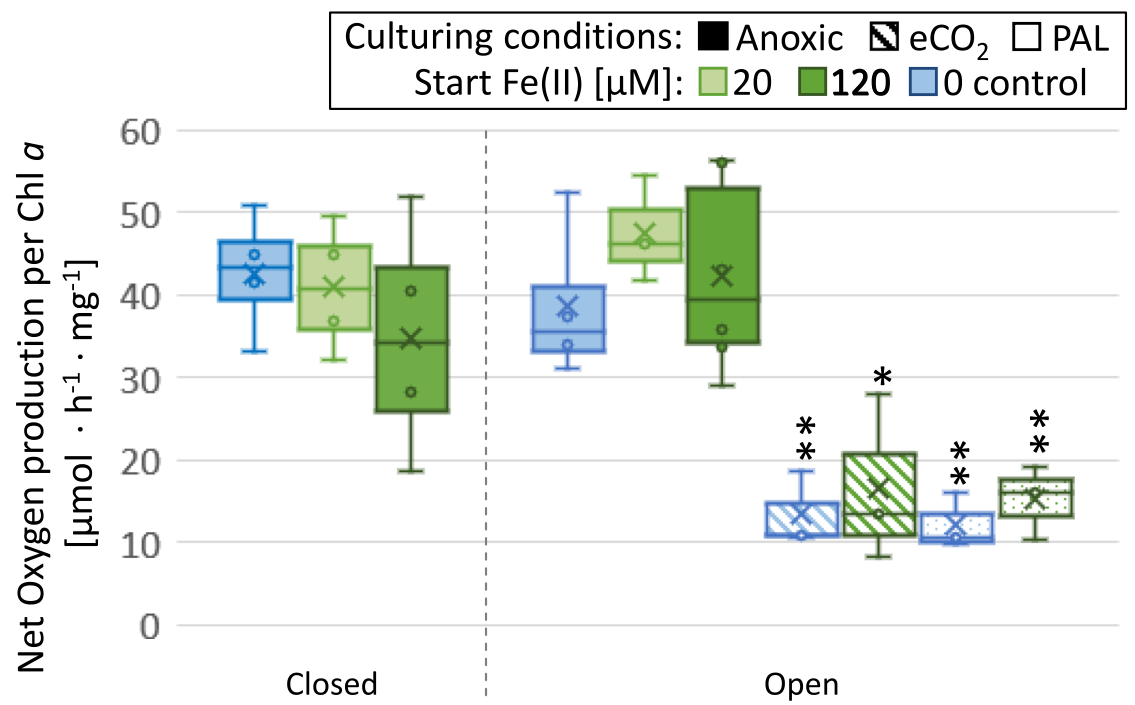

Fig. 5 Boxplot of the hourly net oxygen production rates of Pseudanabaena sp. PCC7367 per unit of cellular Chl $a$ content when grown under different atmospheres. The anoxic atmospheres (solid colour) tested were either $\mathrm{N}_{2}$ with $10 \% \mathrm{CO}_{2}$ (closed, left) or $0.2 \% \mathrm{CO}_{2}$ (open, right). The different Fe(II) starting concentrations were $20 \mu \mathrm{M} \mathrm{Fe}$ (II) (light green), $120 \mu \mathrm{M} \mathrm{Fe}$ (II) (dark green) or none for the control (blue). The control atmospheres were ambient air either with the present-day atmospheric levels of $\mathrm{CO}_{2}$ (PAL, dotted) or supplemented with $0.2 \% \mathrm{CO}_{2}$ (eCO $\mathrm{CO}_{2}$ striped). Oxygen production was determined by measuring the oxygen accumulation in 8-day old cultures (open system and ambient air) and in 5-day old cultures (closed system) with an oxygen sensor in a cuvette and piercing oxygen needle sensor in the closed growth vessel, respectively. The light intensity during the measurement was set at the growth light intensity of 25 PPFD. Asterisks $\left({ }^{*}\right)$ represent a significant difference to the open anoxic system under the same light intensity $\left({ }^{\star} p<0.05\right.$; ${ }^{* \star} p<0.01$; Student's t-test, two-tailed). In the boxplot, the cross indicates the mean, the centre line shows the median value and the bounds of the box indicate the $75^{\text {th }}$ and $25^{\text {th }}$ percentiles, calculated with inclusive median, while the whiskers represent the furthest minimum and maximum measurement outliers. $n=4$ (anoxic, closed), $n=3$ (PAL and $\mathrm{eCO}_{2}$ ). The anoxic, open measurements $(n=6)$ had data excluded from the control and $20 \mu \mathrm{M}$ Fe(II), which had no $\mathrm{O}_{2}$ production, resulting in $n=4$ (control) and $n=3(20 \mu \mathrm{M} \mathrm{Fe}(\mathrm{II})$ ). All measurements were performed on biologically independent samples.

\section{Discussion}

Previous studies have proposed that $\mathrm{Fe}(\mathrm{II})$ at concentrations thought to have existed in the Archaean ocean were toxic to modern-day marine Cyanobacteria, when grown in a closed system with a single addition of $\mathrm{Fe}(\mathrm{II})^{12}$. This toxicity was thought to explain the delay in expansion of Cyanobacteria into the open ocean and initiation of the GOE. Our study used two of the most basal, or phylogenetically deeply rooted marine strains of Cyanobacteria to investigate the effects of $\mathrm{Fe}(\mathrm{II})$ toxicity during the late Archaean. Our data demonstrate that both Cyanobacterial strains still grow well, albeit with two-fold the doubling times of the control cultures (Fig. 3b), if supplemented with a single dose of $\mathrm{Fe}(\mathrm{II})$ at the proposed Archaean ocean concentration of $120 \mu \mathrm{M} \mathrm{Fe}(\mathrm{II})^{23}$. The single time exposure to $\mathrm{Fe}(\mathrm{II})$ did not lead to a significant alteration in net oxygen production after it was oxidised to $\mathrm{Fe}(\mathrm{III})$, neither in the open anoxic, nor in the closed anoxic cultures (Fig. 5). Additionally, net oxygen production rates under anoxic conditions in both culture systems are significantly higher than in control cultures grown under the modern-day oxygen-rich atmosphere (Fig. 5). This increase in $\mathrm{O}_{2}$ release in the anoxic cultures is not due to increased $\mathrm{CO}_{2}$ availability, since oxic control cultures with elevated $\mathrm{CO}_{2}$ did not produce significantly more $\mathrm{O}_{2}$. This experimental result contrasts with the modelled proposed reduction of $36 \%$ in $\mathrm{O}_{2}$ release if toxic $\mathrm{Fe}(\mathrm{II})$ at $25 \mu \mathrm{M}$ is brought to the surface waters ${ }^{12}$, in that we observe the release of $\mathrm{O}_{2}$ during the daytime even with the addition of $120 \mu \mathrm{M} \mathrm{Fe}(\mathrm{II})$ at night (Fig. 3d), comparable to control cultures without $\mathrm{Fe}(\mathrm{II})$ addition. It is therefore unlikely that the toxicity of $\mathrm{Fe}(\mathrm{II})$ at $120 \mu \mathrm{M}$ would have significantly reduced $\mathrm{O}_{2}$ production rates. Significantly, the $\sim 4.5 \mu \mathrm{M}$ concentration of dissolved $\mathrm{O}_{2}$ measured during the daylight period, in rapidly stirred cultures of Pseudanabaena sp. PCC7367 supplemented with $\mathrm{Fe}(\mathrm{II})$ at $120 \mu \mathrm{M}$ at night, simulating the tidal zone, is remarkably close to the modelled $10 \mu \mathrm{M}$, calculated previously for oxygen oases ${ }^{14}$. It could also be shown that GR can form from partially oxidised $\mathrm{Fe}(\mathrm{II})$ in the openculture growth media and its formation process could have negatively impacted Cyanobacterial growth (Fig. 4).

Our data suggest that experiments in closed systems with a single injection of $\mathrm{CO}_{2}$ in the headspace are not suited to investigate the influence of the proposed Archaean environmental conditions on Cyanobacterial growth and oxygen production ${ }^{12}$. Both strains chosen for this study show significantly longer doubling times if incubated in a closed-culture system in comparison to an open-culture system (Fig. 2a, b) or current atmospheric air controls. This is especially evident in the case of Synechococcus PCC7336, which exhibited almost no biological activity in the closed growth system. This reduced growth is already observed prior to when the build-up of oxygen surpasses the background oxygen concentration of the ambient air control cultures at day 8 (Supplementary Fig. 10a). Therefore, the buildup of oxygen observed over the course of the experiment is unlikely to be the cause for the initial reduction in growth. Increased photorespiration under the present atmospheric oxygen levels could be responsible for the observed differences in oxygen production rates between anoxic and oxic growth systems (Fig. 5).

This leaves only the different ambient concentrations of $\mathrm{CO}_{2}$ as a possible explanation for the difference in $\mathrm{Chl} a$ growth between the open- and closed-culture systems, as all other parameters are identical. In the closed-culture system, an atmosphere with $10 \%$ $\mathrm{CO}_{2}$ is initially used to provide $\mathrm{CO}_{2}$ for the whole growth cycle, while the open-growth system permits constant gaseous exchange and maintenance of $\mathrm{CO}_{2}$ levels at $0.2 \%$. The slower growth at $10 \%$ $\mathrm{CO}_{2}$ is not caused by the small drop of $\mathrm{pH}$ in the buffered media from $\mathrm{pH} 7.2$ to $\sim \mathrm{pH} 7$ due to higher levels of dissolved $\mathrm{CO}_{2}\left(\mathrm{CO}_{2}\right.$ (aq) (Supplementary Table 2). However, the increased $\mathrm{CO}_{2(\mathrm{aq})}$ concentration could play a major role in growth inhibition, as 
screening for genes associated with $\mathrm{CO}_{2}$ uptake revealed Synechococcus PCC7336 lacks the pxcA gene (Supplementary Table 3). The PxcA-dependent proton exchange system mediates the cellular $\mathrm{pH}$ homoeostasis by extrusion of protons from the cell during the uptake of $\mathrm{CO}_{2(\mathrm{aq})}$, in which $\mathrm{CO}_{2}$ gets converted to $\mathrm{HCO}_{3}{ }^{-}$and a proton intracellularly ${ }^{27}$. This prevents the acidification of the cytoplasm, as PxcA-independent proton exchange systems are not sufficient to regulate the cellular $\mathrm{pH}$ during $\mathrm{CO}_{2}$ uptake below a media $\mathrm{pH}$ of $8^{28}$. Under present atmospheric levels of $\mathrm{CO}_{2}$, the preferential uptake of $\mathrm{CO}_{2(\mathrm{aq})}$ lasts only for seconds before the small pool of $\mathrm{CO}_{2(\mathrm{aq})}$ is exhausted and $\mathrm{HCO}_{3}{ }^{-}$ becomes the major source of inorganic carbon ${ }^{29}$. But, with the much larger pool of $\mathrm{CO}_{2(\mathrm{aq})}$ under $10 \% \mathrm{CO}_{2}$ conditions in the closed system, the prolonged uptake of $\mathrm{CO}_{2(\mathrm{aq})}$ and subsequent internal acidification could be a major stressor in Synechococcus PCC7336 during growth. Even though Pseudanabaena sp. PCC7367 possesses the $p x c A$ gene, its growth was still reduced under $10 \% \mathrm{CO}_{2}$ conditions. Cyanobacteria possess a carbon concentrating mechanism whereby the levels of $\mathrm{CO}_{2}$ are raised around the enveloped enzyme Ribulose-1,5-bisphosphate carboxylase-oxygenase (RuBisCO) in the carboxysome ${ }^{29}$. Possibly, the $\mathrm{CO}_{2}$ impermeable carboxysome shell acts as a kinetic barrier for the fixation of carbon at $\mathrm{CO}_{2}$ concentrations larger than $3 \%$ by keeping the $\mathrm{CO}_{2}$ on the outside of the carboxysome, rather than concentrating it around the carbon fixing $\mathrm{RuBisCO}$ on the inside ${ }^{30}$.

The $\mathrm{O}_{2}$ production rates obtained in this study can now be taken into consideration when modelling the flux of atmospheric oxygen under Archaean conditions, as a higher primaryproductivity rate per Chl a content would require less biomass and a lower nutrient influx to achieve the same level of oxygen released.

The data from the closed-culture experiment seem to confirm the previously described toxicity of $\mathrm{Fe}$ (II) to Cyanobacteria ${ }^{12}$. Concentrations of $\mathrm{Fe}(\mathrm{II}) \geq 120 \mu \mathrm{M}$ lead to a significant decrease in Chl $a$ growth in Pseudanabaena sp. PCC7367, whereas Synechococcus PCC7336 was unable to grow in this culture system and hence the toxic effects of $\mathrm{Fe}$ (II) could not be assessed (Fig. 2a, b). This was in stark contrast to the results from the open-culture system, where the addition of $\mathrm{Fe}(\mathrm{II})$ up to $120 \mu \mathrm{M}$ led to only a short delay in the growth of Pseudanabaena sp. PCC7367, while Synechococcus PCC7336 showed a long-term reduced Chl a growth when compared to the non-supplemented controls, but with no clear dose-dependent effect. Addition of $\mathrm{Fe}(\mathrm{II})$ to a concentration of $600 \mu \mathrm{M}$ in the open-culture system lead to the precipitation of a green precipitate within 4 days in both strains. ${ }^{57} \mathrm{Fe}$ Mössbauer spectroscopy presented a spectrum that had hyperfine parameters similar to GR, although the additional presence of short-range ordered Fe(III) could not be ruled out (Supplementary Fig. 7 and Supplementary Table 4). This coincided with the arrest of all biological activity, as reflected in a lack of $\mathrm{Fe}(\mathrm{II})$ oxidation and no further increase in Chl $a$ levels in the cultures (Fig. 2). A green precipitate, which we consider to be GR, also formed in the closed-culture system when the atmospheric composition was changed to a lower $\mathrm{CO}_{2}$ concentration of $0.2 \%$ (Supplementary Table 1). The suppression of GR formation at $10 \%$ atmospheric $\mathrm{CO}_{2}$ could be caused by the lower production of $\mathrm{OH}^{-}$ions ${ }^{27}$ during the $\mathrm{Fe}(\mathrm{II})$ oxidation in the early stages of growth. The growth media is buffered to a pH between $6.9(10 \%$ $\left.\mathrm{CO}_{2}\right)$ and $\mathrm{pH} 7\left(0.2 \% \mathrm{CO}_{2}\right)$, but GR formation typically only occurs above $\mathrm{pH} 7.1^{17}$. At low concentrations of $\mathrm{CO}_{2(\mathrm{aq})}$, Cyanobacteria switch from the uptake of $\mathrm{CO}_{2(\mathrm{aq})}$ to the uptake of $\mathrm{HCO}_{3}^{-}$as an inorganic carbon source which releases $\mathrm{OH}^{-}$ ions 29,31 . Therefore, high $\mathrm{CO}_{2(\mathrm{aq})}$ concentrations, as expected of a $10 \% \mathrm{CO}_{2}$ atmosphere, could have delayed the switch to $\mathrm{HCO}_{3}{ }^{-}$ uptake and thus $\mathrm{OH}^{-}$release required to raise the $\mathrm{pH}$ to the level required for GR precipitation. By the time the Cyanobacteria do switch to $\mathrm{HCO}_{3}{ }^{-}$and start to produce $\mathrm{OH}^{-}$, insufficient $\mathrm{Fe}(\mathrm{II})$ remained for the precipitation of GR. These results indicate that the $\mathrm{CO}_{2}$ atmospheric levels might play a crucial role in the formation of GR in a neutral Archaean ocean.

As all available $\mathrm{Fe}(\mathrm{II})$ in the experiments at the proposed Archaean ocean concentration was oxidised within 2 days, the possibility of longer repeated exposure to Fe(II) was explored. Our data showed that the oxidation of Fe(II) only occurred passively by the photosynthetically produced $\mathrm{O}_{2}$ during the light (day) cycle, while dark (nocturnal) Fe(II) levels remain stable (Fig. 3d). Stirring the cultures to inhibit the build-up of $\mathrm{O}_{2}$ and slow the oxidation of $\mathrm{Fe}(\mathrm{II})$ was also explored. Unlike the incomplete oxidation of $\mathrm{Fe}(\mathrm{II})$ in previous experiments at $0.3 \%$ $\mathrm{CO}_{2}{ }^{20}$, all $\mathrm{Fe}$ (II) was completely oxidised during the light cycle at $0.2 \% \mathrm{CO}_{2}$. However, the oxidation rate of the $\mathrm{Fe}(\mathrm{II})$ could be significantly reduced by $36 \pm 15 \%$ by stirring the culture media in comparison to the non-stirred culture (Fig. 3c). Even with mixing, oxygen levels in the media remained steady at $\sim 4.5 \mu \mathrm{M}$, which is in good agreement with modelled Archaean oxygen oases' dissolved $\mathrm{O}_{2}$ concentrations of $1-10 \mu \mathrm{M}^{14}$. Nevertheless, stirring only prolonged the light cycle exposure to $\mathrm{Fe}(\mathrm{II})$ by 10-30 min, depending on the photosynthetic activity of the culture. In order to reduce the Fe(II) oxidation by more than $36 \%$, stronger agitation would be required which would most likely introduce additional shearing stress during a long-term experiment. This is supported by the observation that even a strong single daily shaking event in the continuous $\mathrm{Fe}$ (II) exposure experiment leads to a decrease in growth in the control cultures in comparison to previous experiments (Fig. $3 \mathrm{~b}$ vs Fig. $2 \mathrm{a}, \mathrm{b}$ ). The repeated addition of $\mathrm{Fe}(\mathrm{II})$ overnight for 12 cycles caused a significant reduction in the accumulation of Chl $a$ in both strains (Fig. 3b). During the light cycle, all Fe(II) was rapidly oxidised, which suggests that Cyanobacterial mats could indeed form oxygen oases, as suggested by Farquhar et al. ${ }^{32}$, shielding them from the influence of $\mathrm{Fe}(\mathrm{II})^{12}$.

Oxygen oases could also have released oxygen to the atmosphere locally, as the measured iron oxidation rates are only $16-25 \%$ of the net oxygen production rates on a per mol basis (Figs. 3d and 5). This is supported by evidence from the rock record which show mild oxidative weathering of surface rocks releasing important micronutrients like molybdenum ${ }^{18}$. In the shallow-water coastal regions, the produced Fe(III) could have reacted with the tidal influx of a ferruginous Archaean ocean to form GR, as was observed during the nightly additions of $\mathrm{Fe}(\mathrm{II})$. This may also be the case in the open ocean, with upwelling of $\mathrm{Fe}$ (II) in the water column reacting with $\mathrm{Fe}(\mathrm{III})$ produced during the day to form GR. In an Archaean ocean environment, the produced GR (or Fe(III) precursor) may have been washed into the open ocean and contributed to the formation of BIF as illustrated in Fig. $1^{17}$. Experiments where GR was added directly to exponentially growing cultures of Pseudanabaena sp. PCC7367 showed that pure GR had no significant effect on growth, even at concentrations four-fold higher than the amounts obtained in the open-culturing experiment (Fig. 4a). Most likely, the formation process of GR/Fe(III) hydroxides caused the inhibition of the growth of Pseudanabaena sp. PCC7367, as the complete oxidation of remaining media $\mathrm{Fe}(\mathrm{II})$ and GR precipitate restored growth (Fig. 4a). Light microscopic images show that the oxidation of $\mathrm{Fe}$ (II) in the media by Pseudanabaena sp. PCC7367 lead to the encrustation of cells by the oxidation products (Fig. 4d), whereas the addition of GR resulted in cells adhering to the outside of the GR granule (Fig. 4e) without inhibition of growth (Fig. 4a) or change in growth morphology, as compared to the control (Fig. 4b). Therefore, our data suggest that only the formation of GR, not GR itself, had an inhibitory effect on growth 
(Fig. 4), which could be overcome in cultures with sufficiently high oxygen production to completely oxidise all media Fe(II), thereby preventing their encrustation by newly formed GR/Fe (III) hydroxides (Figs. 3a and 4a). Otherwise, the ongoing formation of GR would lead to a self-reinforcing process, whereby the decreasing photosynthetic activity by GR formation prevents the detoxification of $\mathrm{Fe}(\mathrm{II})$ by oxidation until the culture enters a state of minimal biological activity. A possible mode of GR toxicity could be the potential mechanical stress during the formation of GR on already $\mathrm{Fe}$ (III) encrusted bacterial cells. Therefore, we propose that the widespread colonisation of Archaean shallow-water environments by Cyanobacteria, specifically the multicellular, benthic Pseudanabaena sp. PCC7367 ancestral strain used in this study, was not delayed by the toxicity of $\mathrm{Fe}(\mathrm{II})$, or by the formation of GR under the levels of $\mathrm{Fe}(\mathrm{II})$ thought to have been present in the Archaean ocean. Additionally, our study emphasises the importance of experimental setup, diurnal cycling as well as strain selection (unicellular vs multicellular; benthic vs planktonic) and growth optimisation, in order to gain insight into the events leading up to the oxygenation of the Earth's atmosphere.

\begin{abstract}
Methods
Cultures. Pseudanabaena sp. PCC7367 and Synechococcus sp. PCC7336 were purchased from the Pasteur Culture Collection (PCC) and maintained in ASN-III medium $\left(\mathrm{PPC}^{33}\right)$ on a $16: 8 \mathrm{~h}$ light-dark cycle in a Percival culture chamber (E-22L) at $24^{\circ} \mathrm{C}, 65 \%$ relative humidity under normal atmospheric composition $(400 \pm 20$ ppm $\mathrm{CO}_{2}$ ). Both cultures were grown at $25 \mathrm{PPFD}\left(\mu \mathrm{mol}\right.$ photons per $\mathrm{m}^{2} / \mathrm{s}$ ), the highest light intensity shown not to induce light stress defined by not increasing the carotenoid (CA) over $\mathrm{Chl} a$ ratio (Supplementary Fig. 9). Light intensity in PPFD was measured with a Li-Cor Quantum sensor attached to the Li-250A Light Meter (Li-Cor, USA). Cultures grown under anoxic conditions were maintained in ventilated $\mathrm{T}_{75}$ suspension culture flasks (Sarstedt, Germany) in a self-built anoxic box (construction plans and software available at biorxiv.org/cgi/content/short/ 2020.12.17.423238v1), which was placed inside a culture chamber (Percival E-22L) with the same light and temperature settings as the control cultures. An anoxic atmosphere was retained by constantly removing oxygen generated during photosynthesis with forming gas (Arcal F5, AirLiquide, Germany) on a palladium catalyst (NeoLab Migge, Germany), while $0.2 \%$ of $\mathrm{CO}_{2}$ was maintained by a WMA$4 \mathrm{CO}_{2}$ Analyzer (PP-Systems, USA). Forming gas and $\mathrm{N}_{2}$ inflow as well as $\mathrm{O}_{2}, \mathrm{H}_{2}$ and relative humidity levels were regulated with an Arduino-based control system (Seeeduino lotus, Seeed studio, China). $\mathrm{O}_{2}$ levels $( \pm 6 \mathrm{ppm}$ ) were monitored using a ME2-O2- $\$ 20$ oxygen sensor (Zhengzhou Winsen Electronics, China). Both strains were acclimated to their culture conditions for at least 8 weeks ( 4 transfers) prior to commencing the initial growth curve experiments. A repeat growth curve assessment 6 months later in the anoxic growth chamber Glovebox Mega E-Line 4 (Glovebox Systemtechnik, Germany) did not show any difference in doubling times compared to the self-built anoxic box. The Glovebox anoxic growth chamber uses a $\mathrm{N}_{2}$ atmosphere without added hydrogen, where $\mathrm{O}_{2}$ is constantly removed by a regeneratable copper catalyst. All other environmental parameters, such as $\mathrm{CO}_{2}$ concentration or lighting regime and intensity were exactly replicated as in the selfbuilt anoxic box. $\mathrm{O}_{2}$ levels $( \pm 0.1 \mathrm{ppm})$ in the anoxic growth chamber were monitored using an AutoOxi R 2.01 oxygen trace sensor (Glovebox Systemtechnik, Germany). All experiments that required an anoxic atmosphere after the initial comparison of open-culturing vs closed-culturing growth systems were conducted in the more spacious Glovebox anoxic growth chamber.
\end{abstract}

Comparison of growth systems. For the closed-culture system, $80 \mathrm{ml}$ of ferric ammonium citrate-free ASN-III media, buffered with $22 \mathrm{mM} \mathrm{NaHCO}_{3}$, was dispensed into $100 \mathrm{ml}$ acid-washed, laboratory glass bottles (Schott, Germany) and sealed with butyl rubber stoppers (Zscheile \& Klinger, Germany). The control cultures were set up in standard ASN-III media, buffered with $22 \mathrm{mM} \mathrm{NaHCO}_{3}$.

Sealed media aliquots were made anoxic by gassing for 20 min with $\mathrm{N}_{2}$ gas (99.99\%) before transferring them to the anoxic box for inoculation and $\mathrm{Fe}(\mathrm{II})$ addition. $\mathrm{Fe}(\mathrm{II})$ was added to a final concentration of 20,120 or $600 \mu \mathrm{M}$ in the form of an anoxic, sterile filtered $0.2 \mathrm{M} \mathrm{FeCl}_{2}$ solution in $\mathrm{ddH}_{2} \mathrm{O}$ and the concentrations verified by means of the spectrophotometric ferrozine-based assay. Pseudanabaena sp. PCC7367 and Synechococcus PCC7336 were acclimatised to the anoxic growth conditions for between 2 and 7 months, depending on the experiment conducted. Cultures were inoculated with exponentially growing starter cultures to a final $\mathrm{Chl}$ $a$ concentration of $0.1 \mu \mathrm{g} / \mathrm{ml}$. After inoculation the bottles were sealed, removed from the anoxic box and gassed for $15 \mathrm{~min}$ with Process $\mathrm{CO}_{2} / \mathrm{H}_{2} / \mathrm{N}_{2} 5 / 10 / 85$ (AirLiquide, Germany). The culture vessels were placed on their sides to maximise light and atmospheric exposure in the culture box for 21 days.
In setting up the open-culture system, media was aliquoted into ventilated $\mathrm{T}_{75}$ suspension culture flasks (Sarstedt, Germany), transferred into the anoxic box and equilibrated by daily shaking with the anoxic atmosphere $\left(\mathrm{N}_{2} ; 5 \% \mathrm{H}_{2} ; 0.2 \% \mathrm{CO}_{2}\right.$, $\sim 1 \mathrm{ppm} \mathrm{O}_{2}$ ) for 1 week prior to inoculation. The inoculation and addition of $\mathrm{Fe}(\mathrm{II})$ to this open-culture system was conducted similarly to the closed system experiment. Cultures were incubated within the anoxic box for 21 days at the growth conditions described above.

Cyanobacterial growth and $\mathrm{Fe}$ (II) oxidation in both experimental setups were monitored by Chl $a$ and ferrous iron determinations for 21 days, respectively. The evolution of oxygen inside the closed growth vessels was measured using an OXNP oxygen needle sensor (UNISENSE, Aarhus, Denmark) with a detection limit of $0.3 \mu \mathrm{M}$ dissolved oxygen, which was calibrated according to the manufacturer's instructions. One sensor was permanently inserted in one control culture of Pseudanabaena sp. PCC7367 in buffered ASN-III media for constant monitoring of the dissolved $\mathrm{O}_{2}$ and logged its concentration every $30 \mathrm{~s}$ with the Unisense SensorTrace Suite software (v3.2.000). Dissolved $\mathrm{O}_{2}$ was measured in all closed cultures 10 min before the start of the light cycle and $5 \mathrm{~h}$ after the lights went on, on days $3,5,6,8,14,15$ and 20 .

Chlorophyll $\boldsymbol{a}$ and carotenoid determination. Chl $a$ and CA were extracted in $90 \%(\mathrm{v} / \mathrm{v})$ neutralised methanol and measured as described in Herrmann and Gehringer $^{34}$. The absorption at $780 \mathrm{~nm}$ was used for baseline corrections.

Spectrophotometric ferrozine iron assay. The spectrophotometric ferrozine assay was used to determine the amount of $\mathrm{Fe}(\mathrm{II})$ in the cultures and track its oxidation to Fe(III). Ferrozine (Disodium-4-[3-pyridin-2-yl-6-(4-sulfonatophenyl)1,2,4-triazin-5-yl]benzosulfonate) is a bidentate ligand of metal ions, which binds with Fe(II) forming a brightly purple coloured complex at $562 \mathrm{~nm}^{35}$. Samples inside the anoxic chamber were taken by drawing $500 \mu \mathrm{l}$ of media and precipitating non-soluble components by centrifugation for $1 \mathrm{~min}$ at $15,000 \mathrm{~g}$. Samples from the closed-culture experiments were drawn off with a syringe and immediately added to 1 volume of $2 \mathrm{M} \mathrm{HCl}$ to prevent oxidation during the subsequent $1 \mathrm{~min} 15,000 \mathrm{~g}$ centrifugation step. The following assay was prepared and conducted as described by Viollier et al. ${ }^{36}$. The total reaction volume was reduced to $50 \mu$ f ferrozine solution ( $50 \% \mathrm{w} / \mathrm{v}$ Ammonium acetate, $0.1 \% \mathrm{w} / \mathrm{v}$ Ferrozine in $\mathrm{ddH}_{2} \mathrm{O}$ ) with $200 \mu \mathrm{l}$ sample in order to measure sample absorption at $560 \mathrm{~nm}$ in a 96 -well plate reader (Multiscan FC, ThermoFisher Scientific, USA). Fe(II) calibration solutions were made up fresh from $\mathrm{FeSO}_{4}$ in $1 \mathrm{M} \mathrm{HCl}$. Calibration solutions for total iron determination were prepared by dissolution of high-purity Fe powder $(>99.9 \%)$ in $6 \mathrm{M} \mathrm{HCl}$. In order to determine the total concentration of iron, samples of shaken cultures were added to 1 volume of $2 \mathrm{M} \mathrm{HCl}$ to solubilise finely precipitated solid Fe compounds. $\mathrm{Fe}(\mathrm{III})$ was then reduced to $\mathrm{Fe}(\mathrm{II})$ by the addition of $50 \mu \mathrm{l} 10 \%$ (w/v) hydroxyl hydrochloride in $1 \mathrm{M} \mathrm{HCl}$ to $200 \mu \mathrm{l}$ sample and incubated for $30 \mathrm{~min}$ in a 96-well plate at room temperature prior to the addition of $50 \mu \mathrm{l}$ ferrozine solution.

Fe(II) retention and continuous exposure. In order to assess the amount of $\mathrm{Fe}(\mathrm{II})$ oxidation during the dark cycle, 7-day old, exponentially growing cultures of Pseudanabaena sp. PCC7367 and Synechococcus PCC7336 were used. Cultures and media were prepared and inoculated as for the open-culture experiment and supplemented with $120 \mu \mathrm{M} \mathrm{Fe}(\mathrm{II})$ at the start of the experiment. The cultures were maintained anoxically in a Glovebox Mega E-Line 4 (Glovebox Systemtechnik,

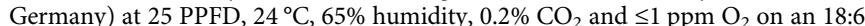
$\mathrm{h}$ light-dark cycle. On day 7 , the cultures were made anoxic by shaking them for $45 \mathrm{~min}$ after the start of the dark cycle and measuring the remaining dissolved oxygen concentration (OX-NP sensor) and Fe(II) levels (ferrozine assay). Sterile $0.2 \mathrm{M} \mathrm{FeCl}_{2}$ solution was added to a final concentration of $120 \mu \mathrm{M}$ and verified by a second ferrozine assay at $5 \mathrm{~min}$ after addition. An OX-NP sensor permanently monitored the dissolved oxygen in one culture flask of each strain. The Fe(II) concentration was measured periodically during the dark cycle and every $10 \mathrm{~min}$ after the start of the light cycle, until no more Fe(II) was detectable, as well as a final measurement of the total $\mathrm{Fe}$ inside the media $150 \mathrm{~min}$ after the lights turned on. As Fe(II) levels remained nearly constant during the dark cycle, this method was used to evaluate the potential toxicity of repeated exposure to $\mathrm{Fe}(\mathrm{II})$, at levels approaching that of the Archaean ocean. Pseudanabaena sp. PCC7367 and Synechococcus PCC7336 were set up as described for the open-culturing system and supplemented every dark cycle to $120 \mu \mathrm{M} \mathrm{Fe}$ (II). The influence of Fe(II) on growth was tracked by daily $\mathrm{Chl} a$ determinations.

The influence of wave mixing on the oxidation of $\mathrm{Fe}(\mathrm{II})$ and the release of oxygen was assessed in a similar manner to the overnight retention experiments. A sterile magnetic stirring bar (442-0487, VWR, USA) was placed into 7-day old exponentially growing cultures of Pseudanabaena sp. PCC7367 during the day cycle and the growth vessel placed on a magnetic stirrer (AAN1.1, Roth, Germany) at $250 \mathrm{rpm}$. After the medium $\mathrm{O}_{2}$ concentration got stabilised, $\mathrm{Fe}(\mathrm{II})$ was added to a concentration of $120 \mu \mathrm{M}$ and its oxidation was determined every 10 min by the ferrozine assay.

Toxicity assessment of green rust. In order to determine the potential toxicity of the GR observed in the open-culture experiments initially provided with $600 \mu \mathrm{M} \mathrm{Fe}$ 
(II), cultures of Pseudanabaena sp. PCC7367 were exposed to different levels of GR during exponential growth. Cultures were prepared and inoculated as for the openculture system experiment. Abiotically produced GR was added on day 4 to a total concentration of either 100,200 or $400 \mu \mathrm{M}$, corresponding to the maximum GR levels that would be produced by the addition of $\mathrm{Fe}(\mathrm{II})$ at $600 \mu \mathrm{M}, 1.2 \mathrm{mM}$ or 2.4 $\mathrm{mM}$, respectively. Care was taken to ensure that no unbound $\mathrm{Fe}(\mathrm{II})$ was added with the GR. Positive controls were made by adding sterile $\mathrm{FeCl}_{2}$ solution to a concentration of $600 \mu \mathrm{M}$ to cultures during inoculation, or in parallel to the addition of GR on day 4. Cultures without the addition of GR or Fe(II) were used as negative controls. The growth was followed by regular $\mathrm{Chl} a$ measurements. Two days after the addition of GR, light microscopic pictures were taken at 100x magnification (Olympus BX53, Japan). On day 9, the Fe(II) and GR in the positive controls were oxidised by removing the cultures from the anoxic chamber followed by gentle shaking under ambient air for $10 \mathrm{~min}$ before being reinserted into the anoxic chamber. Abiotic GR was produced by anoxically adding $\mathrm{FeCl}_{2}$ to $50 \mathrm{ml}$ of $22 \mathrm{mM}$ $\mathrm{NaHCO}_{3}$-buffered ASN-III media to a total concentration of $15 \mathrm{mM}$ and adjusting the medium $\mathrm{pH}$ to 8 through the addition of $0.1 \mathrm{M} \mathrm{KOH}$. This solution was exposed to ambient air while shaking until a shift in colour from initially translucent green to turbid yellow, and finally turbid dark green was observed. The dark green precipitate was collected after centrifugation at $12,000 \mathrm{~g}$ for $10 \mathrm{~min}$ (Centrifuge 5810 R, Eppendorf, Germany) and two subsequent washing steps with anoxic, buffered ASN-III to remove all unreacted $\mathrm{Fe}(\mathrm{II})$. The pellet was suspended in $5 \mathrm{ml}$ buffered ASN-III media and the concentration of the GR as well as its $\mathrm{Fe}$ (II)/Fe(III) ratio was determined by the spectrometric ferrozine assay.

Net oxygen production rates. In order to calculate the net oxygen production rates under different atmospheric conditions, the dissolved oxygen evolution over time was measured by a retractable fibre oxygen minisensor (OXR430, PyroScience, Germany) connected to a MINI-PAM-II (Walz, Germany) inside a constantly stirred measurement cuvette and recorded using the WALZ Wincontrol 3 software V3.28. Cultures were set up as for the open-culture setup in $T_{25}$ ventilated suspension flasks in buffered ASN-III with and without the addition of 120 $\mu \mathrm{M} \mathrm{Fe}(\mathrm{II})$. The oxic cultures were then exposed to normal air with present-day atmospheric levels (PAL) of $\mathrm{CO}_{2}$ or supplemented with $0.2 \% \mathrm{CO}_{2}$. The anoxic cultures in the open-culture system were exposed to an anoxic atmosphere with $0.2 \% \mathrm{CO}_{2}$. Measurements were conducted on 8-day old, mid-exponential phase cultures that were shaken to equilibrate to the growth atmosphere, before $400 \mu$ was transferred into the suspension cuvette (KS-2500, Walz, Germany) of the MINI-PAM-II, which was also situated in the corresponding atmosphere. The Chl a content of $1 \mathrm{ml}$ of culture was determined in parallel. Oxygen measurements were taken for 5 min using the standard growth light irradiance of 25 PPFD under their experimental atmospheric conditions. The difference between the start and end point measurements/readings of dissolved oxygen and the $\mathrm{Chl} a$ concentration was used to calculate the net oxygen production rates per $\mathrm{mg} \mathrm{Chl} a$ per hour. The net oxygen production rate in the closed anaerobic growth vessels was determined from the oxygen measurements taken on day 5 as described in the section 'Comparison of growth systems'.

Statistics and reproducibility. All statistical analyses were done using a twotailed, heteroscedastic Student's $t$-test (Excel 365, Microsoft, USA) to determine the influence of $\mathrm{Fe}(\mathrm{II})$ treatment and the effect of different growth systems on the organisms' growth and $\mathrm{O}_{2}$ production levels. The exact $p$ values are included in the data files for the figures. All experiments were performed once, with measurements taken in parallel from independent biological replicate samples under the same growth conditions.

Mineralogical analysis. In order to determine the composition of the green precipitate, which was formed in the open-culture experiments with an $\mathrm{Fe}$ (II) concentration of $600 \mu \mathrm{M}, 4 \mathrm{ml}$ of shaken culture was centrifuged at $15,000 \mathrm{~g}$ for 15 min and the pellet was washed twice with $\mathrm{ddH}_{2} \mathrm{O}$. The dry pellets were dissolved in $2 \mathrm{M} \mathrm{HCl}$ and the ratio of $\mathrm{Fe}$ (II)-to- $\mathrm{Fe}$ (III) was determined by the ferrozine assay. In order to collect enough sample material for Mössbauer spectroscopy, $400 \mathrm{ml}$ culture was set up in an open-culture system (with $600 \mu \mathrm{M} \mathrm{Fe}(\mathrm{II})$ ) in acid washed, sterile Fernbach flasks with sterile gauze permitting free gaseous exchange. After 1 week, all the precipitate was collected and processed as above, with the addition of a vacuum drying step prior to ${ }^{57} \mathrm{Fe}$ Mössbauer spectroscopic analysis.

The sample was prepared for ${ }^{57} \mathrm{Fe}$ Mössbauer in an anoxic Glovebox $\left(100 \% \mathrm{~N}_{2}\right)$ within which the sample precipitate was sealed inside a plexiglas holder (diameter $=1 \mathrm{~cm}$ ) and stored in an airtight jar at $-20{ }^{\circ} \mathrm{C}$ until measurement. The sample was loaded into a closed-cycle exchange gas cryostat (Janis cryogenics) under a backflow of helium. The measurement was collected at $77 \mathrm{~K}$ with a constant acceleration drive system (WissEL) in transmission mode with a ${ }^{57} \mathrm{Co} / \mathrm{Rh}$ source and calibrated against a $7 \mu \mathrm{m}$ thick $\alpha^{-57} \mathrm{Fe}$ foil measured at room temperature. All spectra were analysed using Recoil V1.05 (University of Ottawa) by applying a Voight-based fitting (VBF) site analysis ${ }^{37}$. The half-width at half-maximum (HWHM) was fixed to $0.125 \mathrm{~mm} / \mathrm{s}$ during fitting.
Growth curve. Maximum doubling times were determined as the inflection of the slope of the growth curve in the exponential phase and used to calculate the doubling time ${ }^{38}$.

Gene screening. The presence of a $p x c A$ gene in Pseudanabaena sp. PCC7367 (GCA_000317065.1) and Synechococcus PCC7336 (GCA_000332275.1) was tested using nBLAST and PSI-BLAST in BLAST+, version 2.10.0 $0^{39}$. Sequences from Synechocystis sp. PCC 6803 were used as reference gen and protein sequence, respectively. The matches with the highest scores are shown in Supplementary Table 3.

Reporting summary. Further information on research design is available in the Nature Research Reporting Summary linked to this article.

\section{Data availability}

Source data for figures (Figs. 2-5 and Supplementary Figs. 8-10) as well as the values for the statistic tests are available on the Zenodo data repository https://doi.org/10.5281/ zenodo.4550675. The authors declare that all other data supporting the findings of this study are available within the paper and its supplementary information files. The gene and protein sequences used in the screen for the $p x c A$ gene (Supplementary Table 3) are accessible through https://www.ncbi.nlm.nih.gov/ under the following links: 14615844, BAA16993.1, CP003592.1 WP_015163468.1, AF448078 and WP_162139143. The construction plans and software for the self-designed anoxic box are available at biorxiv. org/cgi/content/short/2020.12.17.423238v1.

Received: 18 September 2020; Accepted: 26 February 2021; Published online: 06 April 2021

\section{References}

1. Gumsley, A. P. et al. Timing and tempo of the Great Oxidation Event. Proc. Natl Acad. Sci. USA 114, 1811-1816 (2017).

2. Holland, H. D. Volcanic gases, black smokers, and the Great Oxidation Event. Geochim. Cosmochim. Acta 66, 3811-3826 (2002).

3. Lalonde, S. V. \& Konhauser, K. O. Benthic perspective on Earth's oldest evidence for oxygenic photosynthesis. Proc. Natl Acad. Sci. USA 112, 995-1000 (2015).

4. Buick, R. The antiquity of oxygenic photosynthesis: evidence from stromatolites in sulphate-deficient Archaean lakes. Science 255, 74-77 (1992).

5. Flannery, D. T. \& Walter, M. R. Archean tufted microbial mats and the Great Oxidation Event: new insights into an ancient problem. Aust. J. Earth Sci. 59, 1-11 (2012).

6. Heubeck, C. et al. Geological constraints on Archean (3.22 Ga) coastal-zone processes from the Dycedale Syncline, Barberton Greenstone Belt. South Afr. J. Geol. 119, 495-518 (2016)

7. Homann, M., Heubeck, C., Airo, A. \& Tice, M. M. Morphological adaptations of $3.22 \mathrm{Ga}$-old tufted microbial mats to Archean coastal habitats (Moodies Group, Barberton Greenstone Belt, South Africa). Precambrian Res. 266, 47-64 (2015).

8. Crowe, S. A. et al. Atmospheric oxygenation three billion years ago. Nature 501, 535-538 (2013).

9. Farquhar, J., Bao, H. \& Thiemens, M. Atmospheric influence of Earth's earliest sulfur cycle. Science 289, 756-759 (2000).

10. Uyeda, J. C., Harmon, L. J. \& Blank, C. E. A comprehensive study of Cyanobacterial morphological and ecological evolutionary dynamics through deep geologic time. PLoS ONE 11, e0162539 (2016).

11. Sánchez-Baracaldo, P., Raven, J. A., Pisani, D. \& Knoll, A. H. Early photosynthetic eukaryotes inhabited low-salinity habitats. Proc. Natl Acad. Sci. USA 114, E7737-E7745 (2017).

12. Swanner, E. D. et al. Modulation of oxygen production in Archaean oceans by episodes of Fe(II) toxicity. Nat. Geosci. 8, 126-130 (2015).

13. Anbar, A. D. et al. A whiff of oxygen before the Great Oxidation Event? Science 317, 1903-1906 (2007).

14. Olson, S. L., Kump, L. R. \& Kasting, J. F. Quantifying the areal extent and dissolved oxygen concentrations of Archean oxygen oases. Chem. Geol. 362, 35-43 (2013)

15. Cloud, P. E. Significance of the Gunflint (Precambrian) microflora: photosynthetic oxygen may have had important local effects before becoming a major atmospheric gas. Science 148, 27-35 (1965).

16. Cloud, P. Paleoecological significance of the banded iron-formation. Economic Geol. 68, 1135-1143 (1973).

17. Halevy, I., Alesker, M., Schuster, E. M., Popovitz-Biro, R. \& Feldman, Y. A key role for green rust in the Precambrian oceans and the genesis of iron formations. Nat. Geosci. 10, 135-139 (2017). 
18. Kendall, B., Creaser, R. A., Reinhard, C. T., Lyons, T. W. \& Anbar, A. D. Transient episodes of mild environmental oxygenation and oxidative continental weathering during the late Archean. Sci. Adv. 1, e1500777 (2015).

19. Swanner, E. D., Maisch, M., Wu, W. \& Kappler, A. Oxic Fe(III) reduction could have generated Fe(II) in the photic zone of Precambrian seawater. Sci. Rep. 8, 4238 (2018).

20. Rantamäki, S. et al. Oxygen produced by Cyanobacteria in simulated Archaean conditions partly oxidizes ferrous iron but mostly escapesconclusions about early evolution. Photosynth. Res. 130, 103-111 (2016).

21. Fresenborg, L. S., Graf, J., Schätzle, H. \& Schleiff, E. Iron homeostasis of cyanobacteria: advancements in siderophores and metal transporters. In Advances in Cyanobacterial Biology (eds. Singh, P.K., Kumar, A., Singh, V.K. \& Shrivastava, A.K.) 85-117 (Academic Press, 2020).

22. Schirrmeister, B. E., Gugger, M. \& Donoghue, P. C. J. Cyanobacteria and the Great Oxidation Event: evidence from genes and fossils. Palaeontology 58, 769-785 (2015).

23. Canfield, D. E. THE EARLY HISTORY OF ATMOSPHERIC OXYGEN: Homage to Robert M. Garrels. Annu. Rev. Earth Planet. Sci. 33, 1-36 (2005).

24. Krissansen-Totton, J., Arney, G. N. \& Catling, D. C. Constraining the climate and ocean $\mathrm{pH}$ of the early Earth with a geological carbon cycle model. Proc. Natl Acad. Sci. USA 115, 4105-4110 (2018).

25. Catling, D. C. \& Zahnle, K. J. The Archean atmosphere. Sci. Adv. 6, eaax1420 (2020).

26. Moheimani, N. R., Borowitzka, M. A., Isdepsky, A. \& Sing, S. F. Standard methods for measuring growth of algae and their composition in. In Algae for Biofuels and Energy (eds. Borowitzka, M.A. \& Moheimani, N.R.) 265-284 (Springer Netherlands, 2013).

27. Ruby, C., Géhin, A., Abdelmoula, M., Génin, J.-M. R. \& Jolivet, J.-P Coprecipitation of $\mathrm{Fe}$ (II) and $\mathrm{Fe}$ (III) cations in sulphated aqueous medium and formation of hydroxysulphate green rust. Solid State Sci. 5, 1055-1062 (2003).

28. Sonoda, M., Katoh, H., Vermaas, W., Schmetterer, G. \& Ogawa, T. Photosynthetic electron transport involved in PxcA-dependent proton extrusion in Synechocystis sp. Strain PCC6803: effect of pxcA inactivation on CO2, HCO3-, and NO3- uptake. J. Bacteriol. 180, 3799-3803 (1998).

29. Price, G. D., Badger, M. R., Woodger, F. J. \& Long, B. M. Advances in understanding the Cyanobacterial $\mathrm{CO}_{2}$-concentrating-mechanism (CCM): functional components, $\mathrm{Ci}$ transporters, diversity, genetic regulation and prospects for engineering into plants. J. Exp. Bot. 59, 1441-1461 (2008).

30. Moore, K. A. et al. Mechanical regulation of photosynthesis in Cyanobacteria. Nat. Microbiol. 5, 757-767 (2020).

31. Miller, A. G. \& Colman, B. Evidence for $\mathrm{HCO}(3)$ transport by the Blue-Green Alga (Cyanobacterium) Coccochloris peniocystis. Plant Physiol. 65, 397-402 (1980).

32. Farquhar, J., Zerkle, A. L. \& Bekker, A. Geological constraints on the origin of oxygenic photosynthesis. Photosynth. Res. 107, 11-36 (2011).

33. Rippka, R., Stanier, R. Y., Deruelles, J., Herdman, M. \& Waterbury, J. B. Generic assignments, strain histories and properties of pure cultures of Cyanobacteria. Microbiology 111, 1-61 (1979).

34. Herrmann, A. J. \& Gehringer, M. M. An investigation into the effects of increasing salinity on photosynthesis in freshwater unicellular Cyanobacteria during the late Archaean. Geobiology 17, 343-359 (2019).

35. Stookey, L. L. Ferrozine-a new spectrophotometric reagent for iron. Anal. Chem. 42, 779-781 (1970).

36. Viollier, E., Inglett, P. W., Hunter, K., Roychoudhury, A. N. \& van Cappellen, P. The ferrozine method revisited: $\mathrm{Fe}(\mathrm{II}) / \mathrm{Fe}$ (III) determination in natural waters. Appl. Geochem. 15, 785-790 (2000).

37. Rancourt, D. G. \& Ping, J. Y. Voigt-based methods for arbitrary-shape static hyperfine parameter distributions in Mössbauer spectroscopy. Nucl. Instrum. Methods Phys. Res. B 58, 85-97 (1991).
38. Zwietering, M. H., Jongenburger, I., Rombouts, F. M. \& van 't Riet, K. Modeling of the bacterial growth curve. Appl. Environ. Microbiol. 56, 1875-1881 (1990)

39. Altschul, S. F. et al. Gapped BLAST and PSI-BLAST: a new generation of protein database search programs. Nucleic Acids Res. 25, 3389-3402 (1997).

\section{Acknowledgements}

This project was funded by the German Research Foundation, DFG, SPP1833, Grant number: GE2558/3-1 and GE2558/4-1 awarded to MMG. The authors wish to thank Andreas Kappler (University of Tübingen, Germany) for analytical support and Michael Schroda and Julia Lang (Technische Universität Kaiserslautern, Germany) for PAM/O measurements. The authors would like to thank Martin Van Kranendonk and Eva Stüeken for their comments that greatly improved the manuscript.

\section{Author contributions}

A.J.H. and M.M.G. conceptualised the project and designed the research experiments. A.J.H. conducted the experiments and analysed the data. J.S. and J.M.B. performed the ${ }^{57} \mathrm{Fe}-\mathrm{Mössbauer}$ spectroscopy and analysed the data. A.J.H., J.M.B., N.F.-D. and M.M.G. interpreted the data, wrote the manuscript and revised it.

\section{Funding}

Open Access funding enabled and organized by Projekt DEAL.

\section{Competing interests}

The authors declare no competing interests.

\section{Additional information}

Supplementary information The online version contains supplementary material available at https://doi.org/10.1038/s41467-021-22258-1.

Correspondence and requests for materials should be addressed to M.M.G.

Peer review information Nature Communications thanks the anonymous reviewers for their contributions to the peer review reports of the work.

Reprints and permission information is available at http://www.nature.com/reprints

Publisher's note Springer Nature remains neutral with regard to jurisdictional claims in published maps and institutional affiliations.

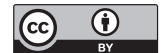

Open Access This article is licensed under a Creative Commons Attribution 4.0 International License, which permits use, sharing, adaptation, distribution and reproduction in any medium or format, as long as you give appropriate credit to the original author(s) and the source, provide a link to the Creative Commons license, and indicate if changes were made. The images or other third party material in this article are included in the article's Creative Commons license, unless indicated otherwise in a credit line to the material. If material is not included in the article's Creative Commons license and your intended use is not permitted by statutory regulation or exceeds the permitted use, you will need to obtain permission directly from the copyright holder. To view a copy of this license, visit http://creativecommons.org/ licenses/by/4.0/.

(C) The Author(s) 2021 